\title{
Comparação entre indivíduos hemiparéticos com e sem histórico de quedas com base nos componentes da Classificação Internacional de Funcionalidade, Incapacidade e Saúde
}

\author{
Comparison between post-stroke hemiparetic subjects with and without history of falls \\ on the basis of the International Classification of Functioning, Disability and Health
}

\author{
Christina Danielli Coelho de Morais Faria ${ }^{1}$, Viviane Amaral Saliba ${ }^{2}$, Luci Fuscaldi Teixeira-Salmela ${ }^{1}$, Sylvie Nadeau ${ }^{3}$
}

Estudo desenvolvido no Depto. de Fisioterapia da UFMG Universidade Federal de Minas Gerais, Belo Horizonte, MG, Brasil

1 Profas. Dras. do Depto. de Fisioterapia da UFMG

2 Fisioterapeuta Ms.

3 Profa. Dra. da Université de Montréal, QC, Canadá

\section{ENDEREÇO PARA}

CORRESPONDÊNCIA

Christina D. C. M. Faria Depto. de Fisioterapia/ UFMG Av. Antônio Carlos 6627 31270-901 Belo Horizonte MG e-mail: cdcmf@ufmg.br

O estudo recebeu apoio da Capes - Coordenação de Aperfeiçoamento de Pessoal de Nível Superior, CNPq - Conselho Nacional de Desenvolvimento Científico e Tecnológico, Fapemig - Fundação de Amparo à Pesquisa do Estado de Minas Gerais, ISB - International Society of Biomechanics, e Governo do Canadá.

ApresentaÇÃO jan. 2010

ACEITO PARA PUBLICAÇÃO maio 2010
Resumo: O objetivo deste estudo foi comparar hemiparéticos com e sem histórico de quedas nos últimos seis meses (caidores e não-caidores) segundo os componentes da Classificação Internacional de Funcionalidade, Incapacidade e Saúde. Dezesseis hemiparéticos não-caidores $(62,3 \pm 16,6$ anos), com tempo de acidente vascular encefálico (AVE) de 38,0 $\pm 48,3$ meses, e 16 caidores $(61,0 \pm 17,0$ anos), tempo de AVE de 48,0 $\pm 50,7$ meses, de ambos os sexos, comunitários e deambuladores, foram avaliados quanto a funções e estruturas do corpo (torque do músculo quadríceps do lado parético e escala de depressão geriátrica), atividade - velocidade de marcha (VM) natural e máxima, teste de levantar e caminhar cronometrado (timed up \& go) e escala de equilíbrio de Berg - e participação (pelo perfil de saúde de Nottingham e escala de qualidade de vida específica para AVE). Os dados foram tratados estatisticamente, com nível de significância $\alpha<0,05$. Não houve diferença significativa entre os grupos quanto à idade, sexo e tempo de AVE $(0,56 \leq p \leq 0,82)$, nem quanto às demais caracteristicas medidas (torque, depressão, VM, equilíbrio e qualidade de vida $(0,12 \leq p \leq 0,60)$. Portanto, hemiparéticos caidores apresentaramse semelhantes aos não-caidores nos domínios funções e estruturas do corpo, atividades e participação. Fatores contextuais ambientais, não analisados, podem estar mais relacionados a episódio(s) de queda nesses indivíduos.

Descritores: Acidente cerebral vascular; Acidentes por quedas; Classificação Internacional de Funcionalidade, Incapacidade e Saúde

ABSTRACT: The aim of this study was to compare post-stroke hemiparetic subjects with and without history of falls over the last six months by using the International Classification of Functioning, Disability and Health (ICF) components. Sixteen community-dwelling hemiparetic subjects without history of falls $(62.3 \pm 16.6$ years, time since stroke $38 \pm 48.3$ months), and 16 with history of fall(s) $(60.9 \pm 17$ years, time since stroke $47.9 \pm 50.8$ months) of both sexes and able to walk, with or without assistive devices, were assessed as to ICF components body functions and structures (torque of the paretic limb quadriceps muscle and depression, by the geriatric depression scale), activities (natural and maximal gait speed, timed up-and-go test and Berg balance scale), and participation (by the Nottingham health profile and the stroke-specific quality of life scale). Groups were statistically compared at significance level $\alpha<0.05$. No statistically significant differences were found between the groups as to age, gender or time since stroke $(0.56 \leq p \leq 0.82)$, neither as to all measured features (torque, depression, gait speed, balance, health-related quality of life $(0.12 \leq p \leq 0.60)$. Therefore, stroke subjects with history of falls were similar to those without history of falls in all measures related to the ICF, suggesting that nonanalysed environmental factors might be more related to falls of hemiparetic poststroke subjects.

Key words: Accidental falls; International Classification of Functioning, Disability and Health; Stroke 


\section{INTRODUÇÃO}

O acidente vascular encefálico (AVE) é definido como uma disfunção neurológica aguda de origem vascular com início rápido dos sintomas, que variam segundo a região afetada do cérebro ${ }^{1}$. Os indivíduos acometidos por AVE desenvolvem incapacidades diversas, que comprometem significativamente os três componentes da Classificação Internacional de Funcionalidade, Incapacidade e Saúde $(\mathrm{CIF})^{2-7}$ - quais sejam, funções e estruturas do corpo, atividade e participação social.

O AVE apresenta elevada prevalência e comumente está associado a elevados índices de morbidade e incapacidade ${ }^{3}$ que resultam em diferentes problemas de saúde pública, dentre os quais as quedas, intercorrências freqüentes, com causas multidimensionais e que geram aumento das co-morbidades e dos riscos de mortalidade ${ }^{8-10}$. Quando comparada à dos idosos, a maior incidência de quedas nos indivíduos acometidos por AVE está ligada a alterações funcionais especificas dessa condição de saúde, como as advindas da hemiparesia ${ }^{8,11}$. Nos hemiparéticos, as quedas comumente ocorrem durante atividades diárias comuns $^{8,10-14}$. Mesmo que alguns desses episódios de quedas não resultem em fraturas, o medo de novas quedas pode restringir a realização de atividades, comprometendo ainda mais a funcionalida$\mathrm{de}^{8-10}$ e gerando conseqüências tão ou até mais prejudiciais do que as fraturas.

Apesar de alguns estudos terem identificado fatores associados ao risco de quedas em hemiparéticos ${ }^{8,10-12,14}$, ainda não foi claramente estabelecido quais são estes fatores e como estão relacionados. Além disso, há pouca informação sobre quedas na população de hemiparéticos brasileiros. Para estes, ainda não foi realizada uma identificação de tais fatores segundo um modelo teórico padronizado e válido, que permita o entendimento mais abrangente de um problema de saúde tão complexo e de causas e conseqüências multidimensionais como as quedas. Nesse contexto, a $\mathrm{CIF}^{2}$ oferece uma estrutura conceitual para a identificação e relação desses fatores, inclusive em hemiparéticos ${ }^{3-5}$ e em idosos com história de quedas ${ }^{15}$. Portanto, o objetivo deste estudo foi comparar hemiparéticos com e sem história de quedas ("caidores" e "não-caidores", respectivamente), considerando os diferentes componentes da CIF.

\section{METODOLOGIA}

Foi desenvolvido um estudo transversal, exploratório, tendo sido recrutados, na comunidade da metrópole de Belo Horizonte, indivíduos com seqüela motora devido ao AVE caracterizando hemiparesia, de ambos os sexos, idade igual ou superior a 20 anos e capacidade de deambulação com ou sem dispositivos de auxílio. Todos já haviam sido tratados ou estavam em tratamento em ambulatórios de reabilitação e/ou hospitais conveniados com universidades ou centros universitários de Belo Horizonte. Foram excluídos indivíduos que não foram capazes de seguir as orientações para a realização dos testes e medidas. A amostra final foi composta de 32 indivíduos. Aqueles que relataram um ou mais episódios de queda nos últimos seis meses foram considerados "caidores" $2,4,6$. Todos assinaram o termo de consentimento aprovado pelo Comitê de Ética em Pesquisa da Universidade Federal de Minas Gerais.

Foram coletados dados antropométricos e clínico-demográficos e, em seguida, a história de quedas foi determinada indagando ao indivíduo e a seu acompanhante a ocorrência de alguma queda nos últimos seis meses. Antes dessa indagação, foi-lhes apresentada uma definição de queda, como recomenda$\mathrm{do}^{16}$, para evitar interpretações ou significados distintos, o que poderia comprometer a validade dos dados coletados $^{16}$

Os testes e medidas utilizados fornecem desfechos já identificados como fatores de risco para quedas ${ }^{4,5}$, tendo sido selecionados com base em estudos prévios $^{4,5}$ que apontaram instrumentos específicos e com propriedades psicométricas adequadas para a avaliação dos componentes da CIF em indivíduos com história de AVE.

No que se refere ao componente funções e estruturas do corpo da CIF, o torque isométrico do músculo quadríceps no membro inferior parético foi avaliado devido à sua importância para a realização de atividades funcionais como a marcha, o passar de sentado para em pé e de em pé para sentado ${ }^{17-19}$, em que as quedas comumente ocorrem ${ }^{8,10-14}$. Foi utilizado um dinamômetro manual digital (Microfetz, USA), segundo protocolo proposto por Andrews et al. ${ }^{20} \mathrm{e}$ Bohannon $^{21}$, que apresenta validade e confiabilidade adequadas ${ }^{21}$. A depressão foi avaliada pela escala de depressão geriátrica $\mathrm{EDG}^{22}$, de 15 itens, dicotômica (respostas sim/não), em que 0 representa possível ausência de depressão e 15 presença. Pontuações totais de 5/6 (nãocaso/caso) são ponto de corte para rastreio de depressão; apresenta adequada validade e confiabilidade e a versão adaptada para o português do Brasil ${ }^{22}$ foi aplicada por entrevista.

Quanto ao componente atividades, a velocidade de marcha (VM) natural e máxima ${ }^{23}$ foi aferida por ser uma importante medida de desempenho funcional, com adequada confiabilidade em hemiparéticos ${ }^{5,24}$. Foi utilizado o protocolo proposto por Flansbjer et al. ${ }^{24} \mathrm{e}$ calculada a $\mathrm{VM}(\mathrm{m} / \mathrm{s})$. Se necessário, foi permitido o uso de órteses ou dispositivo de auxílio à marcha. Foram realizadas três repetições em cada velocidade e análise da média ${ }^{24}$. O teste de levantar e sentar cronometrado TLCC (TUG, na sigla em inglês de timed up \& go $)^{13}$ apresenta adequadas propriedades psicométricas em indivíduos com história de AVE e abrange importantes atividades do dia-a-dia que são consideradas de grande risco de quedas ${ }^{13,19}$; consiste em levantar-se de uma cadeira, andar 3 $\mathrm{m}$ e retornar à cadeira. Para aplicar o TLCC foi utilizado o protocolo proposto por Podsiadlo et al. ${ }^{13}$. Foi medido o tempo médio (s) de três repetições com cronômetro digital ${ }^{24}$. Tempo no TLCC igual ou superior a 14 segundos é um indicativo de maior risco de quedas ${ }^{13,25}$. A escala de equilíbrio de Berg (EEB) ${ }^{26}$ avalia o equilíbrio durante o desempenho de tarefas funcionais, como alcance e transferências. É constituída por 14 itens pontuados numa escala de 0 a 4 (máximo de 56 pontos), na qual 0 representa inabilidade ou necessidade de máxima assistência para completar a tarefa, e 4 indica independência e segurança na realização da tarefa. Foi utilizada a versão adaptada 
para o português do Brasil, com adequadas propriedades psicométricas ${ }^{26}$.

Finalmente, para avaliar o componente participação, foram administrados dois instrumentos de avaliação da qualidade de vida relacionada à saúde (QVRS), sob a forma de entrevista. O perfil de saúde de Nottingham (PSN) ${ }^{27}$, instrumento genérico de QVRS, válido e confiável, é constituído por 38 itens dicotômicos (respostas sim/não). Para a pontuação do instrumento foi considerado o número de respostas "não", com pontuação máxima de 38 referente à melhor, e a mínima de 0 , à pior percepção de qualidade de vida. Foi utilizada a versão adaptada para o português do Brasil ${ }^{27}$. A escala de qualidade de vida específica para AVE (EQVE-AVE) ${ }^{28}$ é um instrumento específico e válido de avaliação da QVRS de indivíduos que sofreram AVE, constituída por 49 itens. Em cada item há cinco opções de respostas, sendo a pontuação mínima igual a 49 (maior dependência e dificuldade nas tarefas diárias) e máxima igual a 245 (melhor percepção de qualidade de vida). Foi utilizada a versão adaptada para o português do Brasil ${ }^{28}$.

Estatística descritiva e testes de normalidade foram realizados para todas as variáveis pelo programa SPSS (v.15.0), seguindo as orientações de Portney $e$ Watkins $^{29}$. O teste $\mathrm{t}$ de Student para amostras independentes, ou o de MannWhitney, ou o do qui-quadrado, ou o teste exato de Fisher foram utilizados para comparar quanto às medidas selecionadas para avaliar os componentes da CIF. Considerando o número de comparações realizadas entre os grupos, foi aplicada a correção de Bonferroni29 $(\alpha=0,006)$.

\section{RESULTADOS}

Foram avaliados 32 indivíduos hemiparéticos cujas características clínicodemográficas, de acordo com a história de quedas, são apresentadas na Tabela 1 . Dezesseis deles, com média de idade de 62,3 anos e com tempo médio de AVE de 38 meses, não relataram episódio de queda nos seis meses anteriores, constituindo o grupo de não-caidores. Outros 16 ,
Tabela 1 Características clínico-demográficas dos grupos de caidores e nãocaidores e valores de $p$ da comparação entre os grupos

\begin{tabular}{|c|c|c|c|c|c|}
\hline \multicolumn{2}{|l|}{ Característica } & $\begin{array}{c}\text { Não-caidores } \\
(n=16)\end{array}$ & $\begin{array}{c}\text { Caidores } \\
(n=16)\end{array}$ & $\begin{array}{c}\text { Teste } \\
\text { estatístico }\end{array}$ & $p$ \\
\hline \multicolumn{2}{|l|}{ Idade (anos) } & $62,3 \pm 16,6$ & $60,9 \pm 17$ & t de Student & 0,82 \\
\hline \multirow{2}{*}{ Sexo } & \multirow{2}{*}{$\begin{array}{l}\text { Masculino } \\
\text { Feminino }\end{array}$} & 8 & 9 & \multirow{2}{*}{$\begin{array}{c}\text { Qui- } \\
\text { quadrado }\end{array}$} & \multirow{2}{*}{0,73} \\
\hline & & 8 & 7 & & \\
\hline \multicolumn{2}{|c|}{ Tempo de AVE (meses) } & $38,0 \pm 48,3$ & $47,9 \pm 50,8$ & Mann-Whitney & $y 0,56$ \\
\hline \multirow[t]{2}{*}{ Classificação } & judo ( $<6$ meses) & 6 & 7 & \multirow{2}{*}{$\begin{array}{l}\text { Exato de } \\
\text { Fisher }\end{array}$} & \multirow{2}{*}{0,72} \\
\hline & Crônico ( $\geq 6$ meses) & 10 & 9 & & \\
\hline \multirow[t]{2}{*}{ Número de AVE } & 1 & 16 & 14 & \multirow{2}{*}{$\begin{array}{l}\text { Exato de } \\
\text { Fisher }\end{array}$} & \multirow{2}{*}{0,48} \\
\hline & + de 1 & 0 & 2 & & \\
\hline \multirow[t]{2}{*}{ Tipo de AVE } & Isquêmico & 11 & 12 & \multirow{2}{*}{$\begin{array}{l}\text { Exato de } \\
\text { Fisher }\end{array}$} & \multirow{2}{*}{0,50} \\
\hline & Hemorrágico & 5 & 4 & & \\
\hline \multirow{2}{*}{ Reabilitação motora } & Já fez/faz & 15 & 10 & \multirow{2}{*}{$\begin{array}{l}\text { Exato de } \\
\text { Fisher }\end{array}$} & \multirow{2}{*}{0,50} \\
\hline & Nunca fez & 1 & 6 & & \\
\hline
\end{tabular}

$\mathrm{AVE}=$ acidente vascular encefálico

com média de idade de 60,9 anos e tempo médio de AVE de 47,9 meses, relataram algum episódio de queda nos últimos seis meses, constituindo o grupo de caidores. Como mostra a Tabela 1, não houve diferença estatisticamente significativa entre os grupos para qualquer das variáveis clínico-demográficas.

Os valores de tendência central e dispersão das variáveis relacionada aos componentes da CIF estão apresentadas na Tabela 2. Novamente, como mostra a tabela, para todas as variáveis dos componentes da CIF não foram encontradas diferenças estatisticamente significativas entre os grupos.

DISCUSSÃO

Esse estudo comparou diferentes variáveis com potencial de estarem relacionadas com episódios de queda em hemiparéticos, divididos em grupos com ou sem história de queda(s) nos últimos seis meses, considerando componentes da CIF. Segundo os resultados, os hemiparéticos com história de queda(s) apresentaram-se semelhantes àqueles sem história de queda(s) nos últimos seis meses tanto nas características clínicodemográficas quanto nas medidas e escores de características dos componentes da CIF.

Tabela 2 Torque do quadriceps, velocidade da marcha (VM), tempo no TLCC (média \pm desvio padrão) e escores dos grupos não-caidores e caidores nos testes de equilíbrio, depressão e qualidade de vida (mediana e diferença interquartis); valor de $p$ da comparação entre os grupos

\begin{tabular}{|c|c|c|c|c|c|}
\hline Medidas / Escores & \multicolumn{2}{|c|}{ Não-caidores $(n=16)$} & \multicolumn{2}{|c|}{ Caidores $(n=16)$} & $p$ \\
\hline Torque do quadríceps (N.m) & \multicolumn{2}{|c|}{$70,36 \pm 28,28$} & \multicolumn{2}{|c|}{$60,81 \pm 27,59$} & 0,34 \\
\hline VM natural (m/s) & \multicolumn{2}{|c|}{$0,82 \pm 0,29$} & \multicolumn{2}{|c|}{$0,65 \pm 0,31$} & 0,12 \\
\hline VM máxima (m/s) & \multicolumn{2}{|c|}{$1,08 \pm 0,41$} & \multicolumn{2}{|c|}{$0,86 \pm 0,45$} & 0,15 \\
\hline \multirow[t]{2}{*}{$\operatorname{TLCC}(\mathrm{s})$} & \multicolumn{2}{|c|}{$21,5 \pm 20,8$} & \multicolumn{2}{|c|}{$25,4 \pm 19,0$} & 0,37 \\
\hline & Mediana & $\neq$ quartis & Mediana & $\neq$ quartis & \\
\hline Equilíbrio (EEB: mín 0; máx 56) & 52 & 5 & 50 & 6 & 0,38 \\
\hline Depressão (EDG: mín 0; máx 10) & 5 & 7 & 8 & 8 & 0,13 \\
\hline PSN (mín 0; máx 38) & 32 & 13 & 26 & 11 & 0,13 \\
\hline EQVE-AVE (mín 49; máx 245) & 195 & 56 & 180 & 51 & 0,6 \\
\hline
\end{tabular}

TLCC = teste de levantar e caminhar cronometrado; $\mathrm{EEB}=$ escala de equilíbrio de Berg; mín = mínimo; máx =máximo; EDG = escala de depressão geriátrica; $\mathrm{PSN}$ = perfil de saúde de Nottingham; EQVE-AVE= Escala de qualidade de vida específica para acidente vascular encefálico 
O torque do músculo quadríceps do membro parético ${ }^{3,30}$ é uma medida importante do componente de funções e estruturas do corpo e para o desempenho de atividades comuns de ocorrência das quedas ${ }^{8,10-14,19}$. Entretanto, o resultado da medida do torque não foi diferente entre os grupos. Resultados semelhantes foram reportados por Pang et al. ${ }^{31}$, que ao compararem hemiparéticos caidores e não caidores (um ou mais episódio de queda e nenhum episódio de queda nos últimos 12 meses, respectivamente) e não encontraram diferença estatisticamente significativa para a força isométrica dos músculos extensores de joelho do membro inferior parético. Como ressaltado por Pang et al. ${ }^{31}$, apesar da força muscular dos extensores do joelho ser uma medida clínica importante e demonstrar correlação significativa com a realização de diversas atividades funcionais em hemiparéticos ${ }^{30}$, a mesma não parece ser uma medida que diferencie hemiparéticos com e sem história de queda(s).

A depressão também é apontada como associada a um risco maior de quedas, pois pode levar à redução da atenção e/ ou da mobilidade ${ }^{32}$. Neste estudo não foi encontrada diferença entre os grupos na pontuação da EDG. Resultados semeIhantes foram reportados por Hyndman et al. ${ }^{33}$, ao compararem a depressão entre indivíduos caidores (uma ou repetidas histórias de quedas em um período de 12 meses) e não-caidores (sem história de queda no mesmo período), pela escala de ansiedade e depressão hospitalar (hospital anxiety and depression scale); só foi encontrada diferença significativa para a depressão quando compararam aqueles que tinham histórico de múltiplas quedas com os não-caidores ${ }^{33}$. No presente estudo, ambos os grupos foram, em média, classificados no ponto de corte positivo para depressão segundo a $\mathrm{EDG}^{22}$. Provavelmente, as incapacidades advindas com o AVE, independente da história ou não de queda, sejam suficientes para gerar os sintomas de depressão avaliados pela EDG, fazendo com que esta não seja uma variável que diferencie os indivíduos com ou sem história de queda em um período de seis meses. Como sugerido por Hyndman et al. ${ }^{33}$, a depressão pode ser importante para diferenciar hemiparéticos com história de quedas recorrentes, mas incapaz de diferenciar indivíduos que tenham sofrido apenas uma queda. É importante destacar que, apesar de a EDG ter sido desenvolvida para a avaliação de indivíduos idosos, sua utilização em indivíduos hemiparéticos devido ao AVE, mesmo que não sejam idosos, não compromete a informação obtida ${ }^{4}$.

Considerando o componente de atividades, um desfecho importante são as velocidades de marcha natural e máxima, utilizadas para caracterizar o nível funcional de hemiparéticos e refletir mudanças fisiológicas e funcionais ${ }^{24}$. Entretanto, as VM natural e máxima não diferenciaram os grupos. Resultados semelhantes foram reportados por Harris et al. ${ }^{11}$ : a VM natural e máxima não apresentaram diferenças significativas quando indivíduos acometidos pelo AVE sem história de quedas foram comparados àqueles que tinham um ou mais episódios de quedas durante os últimos seis meses. Por outro lado, essa medida pode ser importante para diferenciar indivíduos hemiparéticos com quedas recorrentes ${ }^{4}$.

OTLCC é uma medida de mobilidade funcional envolvendo quatro atividades diferentes e relacionadas a quedas ${ }^{12,13}$. Apesar disso, não foram encontradas diferenças significativas entre os grupos. Belgen et al. ${ }^{14}$ também não reportaram diferença significativa no mesmo teste entre os grupos de hemiparéticos caidores e não-caidores, assim como Pang et al. ${ }^{31}$, que investigaram hemiparéticos caidores (um ou mais episódio de quedas em um período de 12 meses) e não-caidores. Considerando o tempo médio no TLCC neste estudo, ambos os grupos seriam classificados com problemas de mobilidade ${ }^{13}$ e com um risco mais elevado de quedas ${ }^{25}$. Apear de o TLCC ser considerado um teste sensível para diferenciar indivíduos com história de AVE com e sem história de quedas $^{6}$, isto não foi observado neste e em outros estudos ${ }^{14,31}$.

O equilíbrio, desfecho mais associado a quedas ${ }^{11,14}$, também não apresentou diferença significativa entre os grupos. Estudos que investigaram o equilíbrio em associação com a queda em hemiparéticos reportam resultados divergentes. Jorgensen et al. ${ }^{32}$ compararam o equilíbrio (oscilação corporal) entre indivíduos com e sem história de AVE e não encontraram diferenças significativas. Igualmente, Harris et al. ${ }^{11}$, ao avaliarem o equilíbrio pela escala de equilíbrio de Berg, não encontraram diferenças entre indivíduos hemiparéticos que caíram uma ou mais vezes em um período de seis meses e aqueles que não caíram. Finalmente, Pang et al. ${ }^{31}$ afirmaram que o equilíbrio avaliado pela EEB não foi um determinante significativo para explicar queda(s) em um período de 12 meses em hemiparéticos caidores (um ou mais episódio de quedas) quando comparados àqueles sem história de quedas. Por outro lado, Belgen et al. ${ }^{14}$ reportaram que indivíduos na fase crônica do AVE e com múltiplas histórias de quedas apresentaram pontuação na EEB significativamente menor que indivíduos com apenas um episódio de queda, e que estes últimos foram semelhantes a indivíduos sem história de queda. Segundo a escala de Berg, em idosos, pontuações menores que 45 indicam alterações do equilíbrio ${ }^{25}$. Nos resultados deste estudo, em média, ambos os grupos tiveram pontuação acima de 49, o que pode justificar a ausência de diferença significativa no equilíbrio de hemiparéticos caidores e não-caidores.

Apesar de qualidade de vida e participação serem conceitos diferentes, há uma inter-relação entre os mesmos ${ }^{4,5}$, sendo os questionários de QVRS as ferramentas disponíveis e utilizadas para avaliar a participação segundo a $\mathrm{CIF}^{4,5}$. Foster et al. ${ }^{9}$ relataram que indivíduos com história de AVE que tiveram repetidas quedas em um período de seis meses apresentaram menor atividade social, avaliada pelo PSN, e uma possível explicação foi a de que indivíduos com histórias de quedas restringem sua participação para diminuir o risco de novas quedas. Neste estudo, a participação avaliada tanto pelo PSN quanto pelo EQVE-AVE não diferenciou indivíduos com ou sem história de quedas. No presente estudo, os indivíduos não foram agrupados quanto ao número de episódio de quedas, mas apenas em dois grupos segundo a ocorrência ou não de queda(s) em um período de seis meses. Provavelmente, variáveis investigadas como a depressão, a VM máxima, o equilíbrio e a participação sejam importantes para diferenciar hemiparéticos 
com história de múltiplas quedas daqueles com apenas uma queda, como demonstrado por estudos prévios ${ }^{9,14,33}$. Entretanto, é necessário identificar fatores associados até mesmo a um único episódio de queda para estabelecer programas de saúde assistenciais e preventivos, uma vez que um único episódio parece imputar vulnerabilidade a novos episódios $^{34}$.

Masud et al. ${ }^{35}$ apontaram mais de 400 fatores de risco potenciais para quedas, divididos em intrínsecos e extrínsecos. Os fatores intrínsecos são os mais investigados e dentre eles alguns apresentaram forte correlação com as quedas, como idade elevada, sexo feminino, história de quedas precedentes, fraqueza muscular dos membros inferiores, marcha lenta, equilíbrio diminuído e imobilidade. Apesar dessa forte relação, Fa- brício et al. ${ }^{36}$ reportaram que $54 \%$ das quedas relatadas tinham tido como causa o ambiente inadequado, fator extrínseco. Provavelmente, fatores contextuais ambientais, não avaliados no presente estudo, podem ser as variáveis em potencial para diferenciar hemiparéticos com história de queda(s) em um período de seis meses daqueles sem história de queda. Em idosos, os fatores ambientais já foram associados a quedas ${ }^{36,37}$ e o AVE foi descrito como uma das principais disfunções relacionada com menor capacidade funcional em idosos caidores $^{37}$. Entretanto, em hemiparéticos, esses fatores contextuais ambientais ainda não foram investigados. Os fatores contextuais ambientais não analisados podem estar mais relacionados a quedas nesses indivíduos, devendo ser considerados em estudos futuros. Também é necessário determinar as particularidades associadas à ausência de quedas, ao episódio isolado e aos episódios recorrentes em diferentes períodos de análise com hemiparéticos, o que não foi possível neste estudo devido ao tamanho da amostra.

\section{CONCLUSÃO}

Os hemiparéticos com história de queda(s) nos seis meses anteriores ao estudo apresentaram-se semelhantes aos indivíduos sem história de queda, em medidas relacionadas a funções e estruturas do corpo, atividades e participação, assim como em medidas clínicodemográficas, sugerindo-se que fatores contextuais ambientais poderiam ser potenciais diferenciadores.

\section{REFERÊNCIAS}

1 World Health Organization. Recommendations on stroke prevention, diagnosis, and therapy. Stroke. 1989;20:1407-31

2 Organização Mundial de Saúde. Classificação Internacional de Funcionalidade, Incapacidade e Saúde. São Paulo: Edusp; 2003.

3 Geyh S, Cieza A, Schouten J, Dickson H, Frommelt P, Omar Z, et al. ICF core sets for stroke. J Rehabil Med. 2004;36(Suppl 44):135-41.

4 Salter K, Jutai J, Teasell R, Foley N, Bitensky J, Bayley M. Issues for selection of outcome measures in stroke rehabilitation: ICF participation. Disabil Rehabil. 2005;27(9):507-28.

5 Barak S, Duncan P. Issues in selecting outcome measures to assess functional recovery after stroke. NeuroRx. 2006;3(4):505-24.

6 AHCPR - Agency for Health Care Policy and Research. Post-stroke rehabilitation: clinical guideline 16. Rockville, MD; 1995. Disponível em: http:// www.ncbi.nlm.nih.gov/bookshelf/br.fcgi? book=hsarchive \&part=A27305.

7 Kelly-Hayes M, Robertson J, Broderick J, Duncan P, Hershey L, Roth E, et al. The American Heart Association stroke outcome classification. Stroke. 1998;29(6):1274-80.

8 Cheng P, Wu S, Liaw M, Wong A, Tang F. Symmetrical body-weight distribution training in stroke patients and its effect on fall prevention. Arch Phys Med Rehabil. 2001;82(12):1650-4.
9 Foster A, Young J. Incidence and consequences of falls due to stroke: a systematic inquiry. British Med J. 1995;311:83-6.

10 Mackintosh S, Hill K, Dodd K, Goldie P, Culham E. Balance score and a history of falls in hospital predict recurrent falls in the 6 months following stroke rehabilitation. Arch Phys Med Rehabil. 2006;87(12):1583-9.

11 Harris J, Eng J, Marigold D, Tokuno C, Louis C. Relationship of balance and mobility to fall incidence in people with chronic stroke. Phys Ther. 2005;85(2):150-8.

12 Andersson AG, Kamwendo K, Seiger A, Appelros P. How to identify potential fallers in a stroke unit: validity indexes of 4 test methods. J Rehabil Med. 2006;38(3):186-91.

13 Podsiadlo D, Richardson S. The timed "up \& go": a test of basic functional mobility for frail elderly persons. J Am Geriatr Soc. 1991;39(2):142-8.

14 Belgen B, Beninato M, Sullivan P, Narielwalla K. The association of balance capacity and falls self-efficacy with history of falling in community-dwelling people with chronic stroke. Arch Phys Med Rehabil. 2006;87(4):554-61.

15 Gladman J. The International Classification of Functioning, Disability and Health and its value to rehabilitation and geriatric medicine. J Chin Med Assoc. 2008;71(6):275-8. 


\section{Referências (cont.)}

16 Zecevic A, Salmoni A, Speechley M, Vandervoot A. Defining a fall and reasons for falling: comparisons among the views of seniors, health care providers, and the research literature. Gerontologist. 2006;46(3):367-76.

17 Cheng P, Liaw M, Wong W, Tang F, Lee M, Lin P. The sitto-stand movement in stroke patients and its correlation with falling. Arch Phys Med Rehabil. 1998;79(9):1043-6.

18 Engardt M, Knutsson E, Jonsson M, Sternhag M. Dynamic muscle strength training in stroke patients: effects on knee extension torque, electromyographic activity, and motor function. Arch Phys Med Rehabil. 1995;76(5):419-25.

19 Flansbjer U, Downham D, Lexell J. Knee muscle strength, gait performance, and perceived participation after stroke. Arch Phys Med Rehabil. 2006;87(7):974-80.

20 Andrews A, Thomas M, Bohannon R. Normative values for isometric muscle force measurements obtained with hand-held dynamometers. Phys Ther. 1996;76(3):248-59.

21 Bohannon R. Measuring knee extensor muscle strength. Am J Phys Med Rehabil. 2001;80(1):13-8.

22 Almeida O, Almeida S. Confiabilidade da versão brasileira da escala de depressão em geriatria (GDS) versão reduzida. Arq Neuropsiquiatr. 1999;57(2b):421-6.

23 Salbach N, Mayo N, Higgins J, Ahmed S, Finch L, Richards C. Responsiveness and predictability of gait speed and other disability measures in acute stroke. Arch Phys Med Rehabil. 2001;82(9):1204-12.

24 Flanbsjer U, Holmback A, Downham D, Patten C, Lexell J. Reliability of gait performance tests in men and women with hemiparesis after stroke. J Rehabil Med. $2005 ; 37(2): 75-82$.

25 Shumway-Cook A, Baldwin M, Polissar N, Gruber W. Predicting the probability for falls in communitydwelling older adults. Phys Ther. 1997;77(8):812-9.

26 Miyamoto S, Lombardi Jr I, Berg K, Ramos L, Natour J. Brazilian version of the Berg balance scale. Braz J Med Biol Res. 2004;37(9):1411-21.
27 Teixeira-Salmela L, Magalhães L, Souza A, Lima M, Magalhães R, Goulart F. Adaptação do Perfil de Saúde de Nottingham: um instrumento simples de avaliação da qualidade de vida. Cad Saude Publica. 2004;20(4):905-14.

28 Lima R, Teixeira-Salmela L, Magalhães L, Gomes-Neto M. Propriedades psicométricas da versão brasileira da escala de qualidade de vida específica para acidente vascular encefálico: aplicação do modelo Rasch. Rev Bras Fisioter. 2008;12(2):149-56.

29 Portney L, Watkins M. Foundations of clinical research: applications to practice. 2nd ed. New Jersey: PrenticeHall; 2000.

30 Bohannon R. Measurement, nature, and implications of skeletal muscle strength in patients with neurological disorders. Clin Biomech (Bristol, Avon). 1995;10(6):283-92.

31 Pang M, Eng J. Fall-related self-efficacy, not balance and mobility performance, is related to accidental falls in chronic stroke survivors with low bone mineral density. Osteoporos Int. 2008;19(7):919-27.

32 Jongensen L, Engstad T, Jacobsen B. Higher incidence of falls in long-term stroke survivors than in population controls: depressive symptons predict falls after stroke. Stroke. 2002;33(2):542-7.

33 Hyndman D, Ashburn A, Stack E. Fall events among people with stroke living in the community: circumstances of falls and characteristics of fallers. Arch Phys Med Rehabil. 2002;83(2):165-70.

34 Perracini M, Ramos L. Fall-related factors in a cohort of elderly community residents. Rev Saude Publica. 2002;36(6):709-16.

35 Masud T, Morris R. Epidemiology of falls. Age Ageing. 2001;30(Suppl 4):3-7.

36 Fabrício SCC, Rodrigues RAP, Costa Jr ML. Causas e conseqüências de quedas de idosos atendidos em hospital público. Rev Saude Publica. 2004;38(1):93-9.

37 Ferreira FR. Perfil e capacidade funcional do idoso após internação hospitalar em decorrência de queda no município de Belo Horizonte [dissertação]. Belo Horizonte: Universidade Federal de Minas Gerais; 2006. 